\title{
Synergistic effects of combined treatment with simvastatin and exemestane on MCF-7 human breast cancer cells
}

\author{
YUANYUAN SHEN $^{1}$, YINGYING DU ${ }^{1}$, YING ZHANG ${ }^{2}$ and YUEYIN PAN ${ }^{1}$ \\ ${ }^{1}$ Department of Oncology, The First Affiliated Hospital of Anhui Medical University, Hefei, Anhui 230022; \\ ${ }^{2}$ Department of Geriatrics, The Third Affiliated Hospital of Anhui Medical University, Hefei, Anhui 230061, P.R. China
}

Received May 12, 2014; Accepted January 27, 2015

DOI: $10.3892 / \mathrm{mmr} .2015 .3406$

\begin{abstract}
Breast cancer is associated with high levels of incidence, morbidity and mortality; therefore, the identification of effective chemopreventive strategies is crucial. It is important for clinicians to be able to identify the populations at risk who would benefit from chemoprevention, and the interventions that are effective and safe. The aim of the present study was to investigate the combined effects of simvastatin and exemestane on MCF-7 human breast cancer cells. The anti-proliferative effects of simvastatin and exemestane, alone and in combination, on the growth of MCF-7 human breast cancer cells were assessed by MTT assay. The synergism between the two drugs was determined in vitro using the combination index (CI) analysis. Cell cycle distribution and apoptosis were analyzed by flow cytometry, and alterations to the signaling pathway in MCF-7 cells were examined by immunoblotting following treatment with various regimens. The results of the MTT assay indicated that the combined treatment of simvastatin and exemestane significantly decreased the viability of MCF-7 estrogen receptor-positive (ER+) human breast cancer cells, as compared with those that were treated with the individual drugs $(\mathrm{CI}<1)$. In addition, coadministration of exemestane and simvastatin was shown to result in marked inhibition of tumor cell proliferation, significant cell cycle arrest at $\mathrm{G}_{0} / \mathrm{G}_{1}$ phase and induction of apoptosis, as compared with that of the control and individual drug-treated cells. Furthermore, the results of the present study indicated that these synergistic effects may be associated with the B-cell lymphoma 2 (Bcl-2)/Bcl-2-associated $\mathrm{X}$ protein apoptotic pathway and the mitogen-activated protein kinase/mammalian target of rapamycin/p70S6 kinase growth pathway. The combination of exemestane and simvastatin generated synergistic effects on MCF-7 ER+ breast cancer cells, indi-
\end{abstract}

Correspondence to: Professor Yueyin Pan, Department of Oncology, The First Affiliated Hospital of Anhui Medical University, 218 Ji Xi Road, Hefei, Anhui 230022, P.R. China E-mail: panyueyin1965.11@gmail.com

Key words: synergistic effect, combination treatment, simvastatin, exemestane, MCF-7 cating that the combination of these drugs may be a potential therapeutic strategy for the treatment of hormone-dependent breast cancer. The combination of the two inhibitors markedly increased the efficacy, as compared with the single-agent treatment, suggesting that combination treatment could become a highly effective approach for breast cancer. The results of the present study suggested that this combination of drugs has therapeutic potential, and requires further mechanistic and biomarker investigations in clinical trials.

\section{Introduction}

Breast cancer is the most prevalent type of cancer diagnosed in the worldwide female population (1). In patients with breast cancer, $\sim 60 \%$ of pre-menopausal and $\sim 75 \%$ of post-menopausal females have hormone-dependent (estrogen receptor positive $[\mathrm{ER+}]$ ) carcinomas (2), and are therefore suitable for endocrine therapy, which is a therapeutic strategy that aims to suppress the mitogenic effects of estrogen on breast cancer cells (3). There are various types of hormonal therapies that may be used to treat ER+ breast cancers. Recently, aromatase inhibitors (AIs) have been considered the primary choice for hormonal treatment of ER $\alpha+$ breast cancer in postmenopausal females (4). Third-generation AIs include the non-steroidal triazole derivatives, anastrozole and letrozole, which act as competitive inhibitors. Furthermore, a steroidal derivate of androstenedione, exemestane, has been shown to be an effective alternative to tamoxifen. Previous studies have demonstrated that exemestane is superior to tamoxifen, with regards to its effects on disease progression, incidences of locoregional and distant relapses, and contralateral breast cancers $(2,5)$. However, despite advances in breast cancer treatment, $25-40 \%$ of patients will eventually develop metastatic disease, which is largely incurable (6). Systemic chemotherapy is currently considered the standard treatment for patients with metastatic breast cancer (7). In addition, despite the success of the most recent generation of AIs, the eventual occurrence of adverse effects, including bone loss, fractures (8) and acquired resistance (9), reinforce the importance of searching for novel potent and specific agents with lower side effects, which may reverse the acquired resistance and extend the benefits of AIs.

Statins are one of the most frequently prescribed medications, which are used to decrease the risk of cardiovascular events and overall mortality (10). Statins are known to 
decrease high blood cholesterol levels through suppression of hepatic cholesterol biosynthesis (11). Statins have a similar structure to that of 3-hydroxy-3-methylglutaryl-coenzyme A (HMG-CoA) and competitively inhibit HMG-CoA reductase, an enzyme that catalyzes the rate-limiting step in cholesterol biosynthesis, the conversion of HMG-CoA to mevalonate (12). As well as producing cholesterol, the mevalonate pathway produces numerous non-sterol products, including ubiquinone, dolichol, isopentenyladenine and prenyl groups, which are essential for the isoprenylation of intracellular second messenger mitogenic signaling proteins, such as Ras and other small $\mathrm{G}$ proteins (12). These non-sterol isoprenoid byproducts are important regulators of numerous oncogenic properties, including angiogenesis, proliferation and migration $(13,14)$. Since mevalonate is synthesized from HMG-CoA, HMG-CoA reductase inhibitors, also known as statins, reduce the entry of mevalonate into the pathway. Previous studies have demonstrated the anti-neoplastic effects of statins in vitro (15-18). Nielsen et al (19) reported that statin use in Danish patients with cancer was associated with reduced cancer-associated mortality in a large observational study that included $>295,000$ patients with cancer.

Simvastatin is the most commonly used lipid-lowering statin drug, which is derived from lovastatin. Simvastatin has been shown to exhibit anti-proliferative and apoptotic activity against numerous types of cancer cell lines, including colon, prostate and breast (16-18). The anti-tumor mechanisms of simvastatin have been investigated and numerous potential underlying mechanisms have been identified, including suppression of downstream signaling of epidermal growth factor receptors, and attenuation of extracellular signal-regulated kinases $1 / 2$, nuclear factor- $\kappa \mathrm{B}$, c-Jun $\mathrm{N}$-terminal kinases, phosphoinositide 3-kinase/Akt (17), generation of reactive oxygen species (20), activation of inducible nitric oxygen species resulting in increased levels of nitric oxide (21), as well as down regulation of B-cell lymphoma 2 (Bcl-2) and activation of Bcl-2-associated $\mathrm{X}$ protein (Bax) (15). Furthermore, a large Danish nationwide prospective cohort study demonstrated that use of simvastatin, a highly lipophilic statin, reduced the recurrence risk by 10 fewer cases per 100 females over 10 years among Danish females with Stage I-III breast cancer (22).

Therapeutic strategies using a combination of drugs in order to enhance the efficacy of cancer treatment have recently garnered attention. The drugs may act together synergistically to inhibit tumor progression through the regulation of various signaling pathways, or reverse the cancer-specific upregulated cell proliferation or evasion of apoptosis (23). The present study chose to evaluate combinations of statin drugs, based upon previously reported cytotoxic experience in breast cancer cell lines $(24,25)$. The present study tested the hypothesis that the combination of simvastatin and exemestane may suppress the growth of ER+ breast cancer, and investigated the effects of combined exemestane and simvastatin treatment on breast cancer cell function, including cell survival, cell cycle, cell apoptosis and alterations in signaling pathways.

\section{Materials and methods}

Cell culture. The MCF-7 human breast cancer cell line was kindly provided by the Laboratory of Molecular Biology of Anhui Medical University (Anhui, China). The cells were purchased from American Type Culture Collection (Manassas, VA, USA) and were cultured in Dulbecco's modified Eagle's medium (DMEM; HyClone Laboratories, Inc., Logan, UT, USA) supplemented with $10 \%$ heat-inactivated fetal bovine serum, $100 \mathrm{U} / \mathrm{ml}$ penicillin, $100 \mu \mathrm{g} / \mathrm{ml}$ streptomycin and $2 \mathrm{mM}$ L-glutamine, which were all purchased from Sijiqing Biological Engineering Materials (Hangzhou, China), and cells were maintained at $37^{\circ} \mathrm{C}$ in a humidified incubator containing $5 \% \mathrm{CO}_{2}$ and $95 \%$ air. The cells were harvested with trypsin-EDTA once they had reached the exponential growth phase.

Reagents and antibodies. Exemestane was provided by Pfizer Inc. (New York, NY, USA) and was dissolved in dimethyl sulfoxide (DMSO; Sigma-Aldrich, St. Louis, MO, USA) at $100 \mathrm{mM}$, in order to produce a stock solution. Simvastatin was purchased from Sigma-Aldrich and was dissolved in DMSO to a stock concentration of $100 \mathrm{mM}$. The drugs were stored at $-20^{\circ} \mathrm{C}$ and diluted with culture medium prior to use. Final concentrations of exemestane were $3.125,6.25,12.5,25,50$ and $100 \mu \mathrm{M}$, and simvastatin were $1.5625,3.125,6.25,12.5,25$ and $50 \mu \mathrm{M}$. The final concentration of DMSO in the DMEM was kept at $<0.1 \%$, and equal amounts of the solvent were added to the control cells. The following primary antibodies were used: Phosphorylated (p)-mitogen-activated protein kinase (MAPK), p-mammalian target of rapamycin (mTOR), mTOR, P70S6 kinase (K), p-P70S6K (Cell Signaling Technology, Inc., Danvers, MA, USA); Bcl-2, Bax and $\beta$-actin (Santa Cruz Biotechnology, Inc., Dallas, TX, USA).

Growth inhibition assay. The anti-proliferative effects of exemestane and simvastatin on the cells were evaluated using an MTT (Sigma-Aldrich) assay. Exponentially growing cells were seeded in 96 -well plates $\left(1 \times 10^{4}\right.$ cells/well). The cells were incubated overnight for cell attachment and recovery. Following treatment with various concentrations of exemestane or simvastatin for $72 \mathrm{~h}, 20 \mu \mathrm{l}$ MTT solution $(5 \mathrm{mg} / \mathrm{ml})$ was added to each well, and the plates were incubated for a further $4 \mathrm{~h}$ at $37^{\circ} \mathrm{C}$. The colored formazan product was dissolved in $150 \mu \mathrm{l}$ DMSO. The 96-well plates were then agitated for $10 \mathrm{~min}$ at room temperature in order to thoroughly dissolve the MTT product. The optical density (OD) of each well was measured at a wavelength of $490 \mathrm{~nm}$ on an ELISA plate reader (Bio-Rad Laboratories, Inc., Hercules, CA, USA). The percentage of inhibited cell growth resulting from each drug was calculated as follows: [(OD490 control cells $^{-O D} 490_{\text {tre }}$ ated cells $/$ OD $\left.490_{\text {control cells }}\right] \times 100 \%$. The half maximal inhibitory concentration $\left(\mathrm{IC}_{50}\right)$ of the drugs was determined as the drug concentration that resulted in 50\% cell growth inhibition, as compared with the growth of the control cells, following $72 \mathrm{~h}$ exposure to the drugs. Six replicate wells were used for each drug concentration. Experiments were repeated at least three times and performed in triplicate.

Measurement of synergy. The anti-proliferative effects of the interaction between exemestane and simvastatin were assessed by measuring the combination-index (CI), a quantitative representation of the pharmacological interaction between two drugs. The combined effect of exemestane and 
simvastatin was assessed using the median effect analysis method, as previously described by Chou and Talalay (26). The two drugs were combined in a fixed ratio of doses, which typically corresponded to $0.125,0.25,0.5,1,2$, and 4 times that of the individual $\mathrm{IC}_{50}$ values. The $\mathrm{CI}$ values of interactions between exemestane and simvastatin were assessed using CompuSyn 1.01 software (ComboSyn, Inc., Paramus, NJ, USA): $\mathrm{CI}<1, \mathrm{CI}=1$, and $\mathrm{CI}>1$ were considered to indicate synergistic, additive and antagonistic effects, respectively (26).

Cell cycle analysis by flow cytometry. Equal numbers of MCF-7 cells (1x10\%/well) were seeded in six-well dishes and were incubated for $24 \mathrm{~h}$ prior to treatment with exemestane, simvastatin or a combination of the two drugs for $72 \mathrm{~h}$. The adhered cells were harvested by trypsinization (Sijiqing Biological Engineering Materials), washed twice with phosphate-buffered saline (PBS; Sigma-Aldrich) and fixed overnight in $70 \%$ ethanol (Sijiqing Biological Engineering Materials) at $4^{\circ} \mathrm{C}$. The ethanol was removed and the cells were washed a further two times with PBS, prior to resuspension in $1 \mathrm{ml}$ propidium iodide/Triton X-100 staining solution [PBS containing $0.1 \%$ Triton X-100 (Sigma-Aldrich), $200 \mu \mathrm{g} / \mathrm{ml}$ RNAse A (Sigma-Aldrich) and $50 \mu \mathrm{g} / \mathrm{ml}$ propidium iodide (Sigma-Aldrich)] in the dark for $30 \mathrm{~min}$. The cell cycle distribution was measured by flow cytometry using a FACScan system equipped with Cell Quest software (BD Biosciences, San Jose, CA, USA). The percentage of cells in the $\mathrm{G}_{0} / \mathrm{G}_{1}, \mathrm{~S}$, and $\mathrm{G}_{2} / \mathrm{M}$ phases was calculated using ModFit $\mathrm{LT}^{\mathrm{TM}} 4.0$ software (Verity Software House, Topsham, ME, USA) in order to determine the cell cycle distribution.

Annexin $V$ assay for the assessment of apoptosis. The cells in the exponential growth phase were plated $\left(1 \times 10^{6}\right.$ cells/well $)$ in six-well plates, allowed to attach overnight and treated with $\mathrm{IC}_{50}$ values of exemestane and simvastatin, either alone or in combination, for $72 \mathrm{~h}$. Following $72 \mathrm{~h}$ of treatment, the adherent and floating cells were collected, washed twice with precooled $\left(4^{\circ} \mathrm{C}\right) \mathrm{PBS}$ and resuspended in $400 \mu \mathrm{l}$ binding buffer. The cells were then incubated with $5 \mu \mathrm{l}$ Annexin V-fluorescein isothiocyanate (BestBio, Shanghai, China) at room temperature in the dark for $15 \mathrm{~min}$, followed by an incubation with $10 \mu \mathrm{l}$ propidium iodide (40 $\mu \mathrm{g} / \mathrm{ml}$; Sigma-Aldrich) at room temperature in the dark for $5 \mathrm{~min}$. Following incubation, the stained cells were analyzed using a FACScan system equipped with Cell Quest software. Untreated cells were used as controls.

Western blot analysis. The MCF-7 cells treated with or without the drugs were washed with ice-cold PBS and scraped into lysis buffer (HyClone Laboratories, Inc.). The lysates were centrifuged at $16,853 \mathrm{x}$ g for $30 \mathrm{~min}$ at $4^{\circ} \mathrm{C}$, and the supernatants were collected. Briefly, the protein concentration of each sample was determined using a Bicinchoninic Acid Protein Assay kit (Beyotime Institute of Biotechnology, Shanghai, China). Equal amounts of protein $(5 \mu \mathrm{l} ; 0.62 \mathrm{mg} / \mathrm{ml})$ from each sample were separated by $10 \%$ SDS-PAGE (HyClone Laboratories, Inc.) and were transblotted to polyvinylidene difluoride (PVDF) membranes (Millipore, Billerica, MA, USA). The membranes were blocked with a solution of PBS containing 5\% milk and $0.1 \%$ Tween 20 (HyClone Laboratories, Inc.) for $2 \mathrm{~h}$. The PVDF membranes were then probed with the following specific primary antibodies: p-MAPK, MAPK, p-mTOR (9208P; rabbit monoclonal), mTOR (2983P; rabbit monoclonal), p70S6K (2708p; rabbit monoclonal) and p-p70S6K (9234P; rabbit monoclonal), which were used at a dilution of 1:1,000 and were purchased from Cell Signaling Technologies, Inc. (Danvers, MA, USA), as well as Bcl-2 (ab32124; rabbit monoclonal), Bax (ab32503; rabbit monoclonal) and $\beta$-actin (ab133626; mouse monoclonal) were used at a dilution of 1:500 in Tris-buffered saline with $0.1 \%$ Tween 20 (TBST) and were purchased from Abcam, (Cambridge, MA, USA) at $4^{\circ} \mathrm{C}$ overnight. Following rinsing with TBST three times, the PVDF membranes were incubated with a horseradish peroxidase-conjugated secondary antibody $(1: 5,000)$ at room temperature for $1 \mathrm{~h}$. Positive bands were detected using Enhanced Chemiluminescence reagents (EMD Millipore, Billerica, MA, USA). $\beta$-actin was used as a loading control.

Statistical analysis. Values are expressed as the mean \pm standard deviation, obtained from at least three independent experiments. Student's t-test and one-way analysis of variance were used to determine the significant differences between the control and treatment groups. Data processing was performed using the SPSS version 16.0 software package (SPSS Inc., Chicago, IL, USA). $\mathrm{P}<0.05$ was considered to indicate a statistically significant difference.

\section{Results}

Exemestane or simvastatin alone inhibit the growth of MCF-7 cells. The anti-proliferative effects of exemestane and simvastatin as single agents on MCF-7 cells were determined using an MTT assay. The MCF-7 cells were treated with various concentrations of exemestane $(3.125-100 \mu \mathrm{M})$ or simvastatin (1.5625-50 $\mu \mathrm{M})$ for $72 \mathrm{~h}$. A dose-dependent decrease in cell viability was observed following treatment with either exemestane or simvastatin. The $\mathrm{IC}_{50}$ values were $28.02 \pm 2.806 \mu \mathrm{M}$ and $10.93 \pm 1.615 \mu \mathrm{M}$ for exemestane and simvastatin respectively, following $72 \mathrm{~h}$ exposure (Fig. 1). Therefore, $28 \mu \mathrm{M}$ exemestane and $11 \mu \mathrm{M}$ simvastatin were used for all of the subsequent experiments.

\section{Synergistic interaction between exemestane and simvastatin in} MCF-7 cells. To investigate the effects of exemestane combined with simvastatin on MCF-7 cells, the cells were exposed to various concentrations of exemestane and simvastatin for $72 \mathrm{~h}$. Combined treatment with the two agents induced increased levels of cell death, as compared with treatment with either exemestane or simvastatin alone. In the cells treated with exemestane and simvastatin concurrently, the CI values were all $<1$, with mean CI values of 0.25 . These results indicated a synergistic interaction between exemestane and simvastatin on the growth inhibition of MCF-7 cells (Fig. 2). In support of this result, photomicrographs demonstrated that treatment with exemestane or simvastatin alone had only a minor effect on the number of MCF-7 cells and their morphology, whereas combined treatment resulted in a marked reduction of cell proliferation after $72 \mathrm{~h}$ of treatment.

Detection of cell cycle distribution using flow cytometry. To elucidate the mechanisms by which exemestane and 

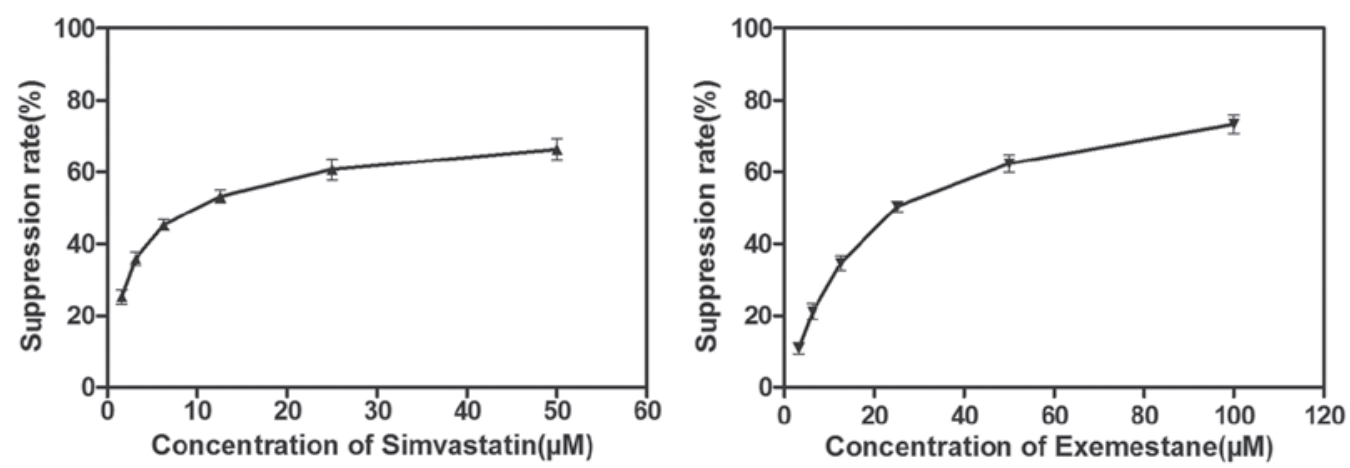

Figure 1. Anti-proliferative effects of various concentrations of exemestane and simvastatin on MCF-7 human breast cancer cells. Dose-response curves were obtained by MTT assay. The cells were exposed to the different concentrations of exemestane (3.125-100 $\mu \mathrm{mol} / \mathrm{l})$ and simvastatin $(1.5625-50 \mu \mathrm{mol} / \mathrm{l})$ for $72 \mathrm{~h}$. Each data point represents the results from at least three independent experiments.

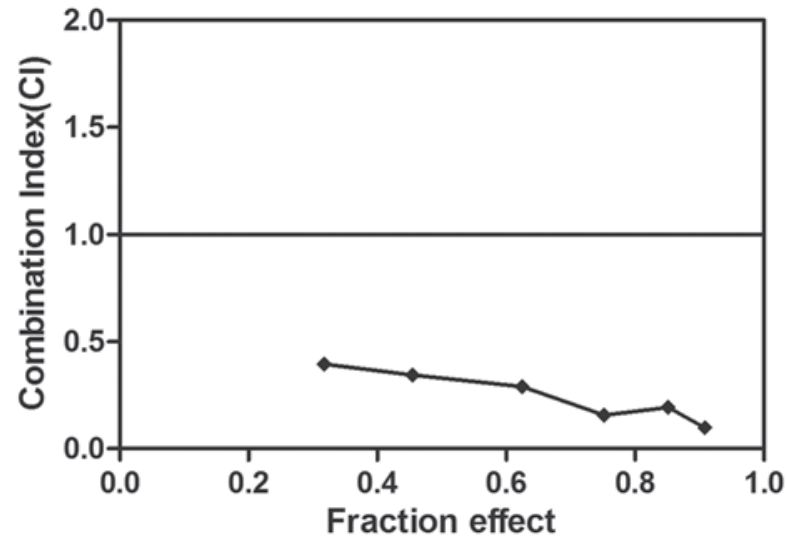

Figure 2. Inhibitory effects of exemestane combined with simvastatin on MCF-7 human breast cancer cells. The CI value was calculated using the Chou-Talalay method, as described previously. The CI value was $<1$ in the MCF-7 cell line following the combined treatment. $\mathrm{CI}<1, \mathrm{CI}=1$ and $\mathrm{CI}>1$ indicate synergistic, additive, and antagonistic effects, respectively. CI, combination index.

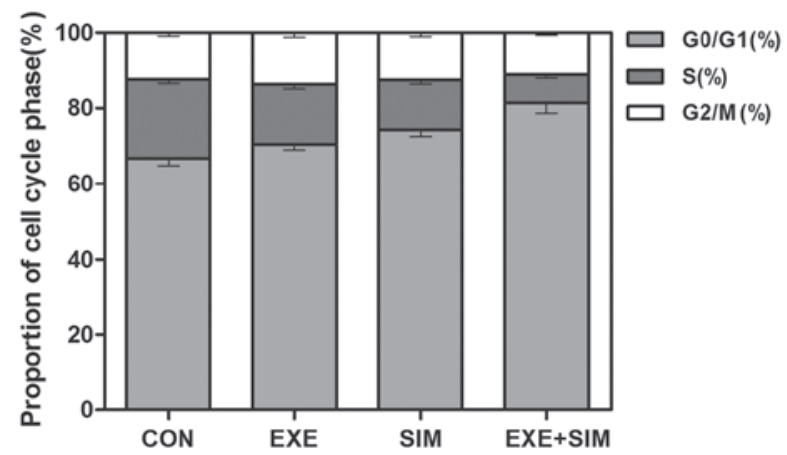

Figure 3. Effects of exemestane combined with simvastatin on the cell cycle distribution of MCF-7 human breast cancer cells measured by flow cytometry. The concentrations of exemestane and simvastatin were used at $\mathrm{IC}_{50}$ levels. Flow cytometric analysis was applied to determine the alterations in cell cycle distribution of MCF-7 cells following treatment with exemestane and simvastatin as single agents, or concurrently for $72 \mathrm{~h}$. CON, control; EXE, exemestane; SIM, simvastatin; EXE+SIM, concurrent administration.

simvastatin inhibit the proliferation of MCF-7 cells, the cell cycle distribution was analyzed by flow cytometry. Treatment with either exemestane or simvastatin increased the population of cells in $\mathrm{G}_{0} / \mathrm{G}_{1}$ phase, with a concomitant decrease of cells in $\mathrm{S}$ phase $(\mathrm{P}<0.05)$ (Fig. 3). In addition, combined treatment with exemestane and simvastatin further increased the percentage of MCF-7 cells in $\mathrm{G}_{0} / \mathrm{G}_{1}$ phase, as compared with the cells treated with either exemestane or simvastatin alone $(\mathrm{P}<0.01)$. These results indicated that these two drugs may exert synergistic growth-inhibitory effects, resulting in a cell cycle arrest in $\mathrm{G}_{0} / \mathrm{G}_{1}$ phase.

Effects of exemestane or simvastatin, either alone or in combination, on cell apoptosis. To examine whether the observed suppression of growth was due to an enhanced rate of apoptosis, the apoptotic rates of the cells treated with exemestane and simvastatin, either alone or in combination, were determined using Annexin V-propidium iodide staining. Annexin V staining is markedly more sensitive for detecting apoptosis, as compared with the methods based on hypodiploid DNA content. Treatment with exemestane combined with simvastatin significantly enhanced apoptosis of the cells, as compared with the treatment with either drug alone. Individual treatment with exemestane and simvastatin resulted in 13.37 and $18.05 \%$ apoptotic cells, respectively, whereas $37.08 \%$ Annexin V-positive cells were observed following combined treatment with the two drugs (Fig. 4). These data indicated that concurrent exposure to exemestane and simvastatin resulted in synergistic interaction in MCF-7 cells.

Exemestane and simvastatin alone or in combination modify the expression levels of mitogen-activated protein kinase (MAPK) and mTOR/p70S6K signaling-associated proteins in MCF-7 cells. The main downstream effect of MAPK activation is inhibition of the mTOR signaling pathways, which have been causally associated with breast cancer cell proliferation, motility and invasiveness. Therefore, the present study analyzed the expression levels of MAPK and mTOR/p70S6 signaling-associated proteins in MCF-7 cells. The combined treatment of simvastatin with exemestane resulted in increased expression levels of p-MAPK and reduced expression levels of p-mTOR and p-p70S6K, whereas the total MAPK, mTOR and p70S6K expression levels were unchanged in response to treatment with simvastatin, exemestane or a combination of the two (Fig. 5). 


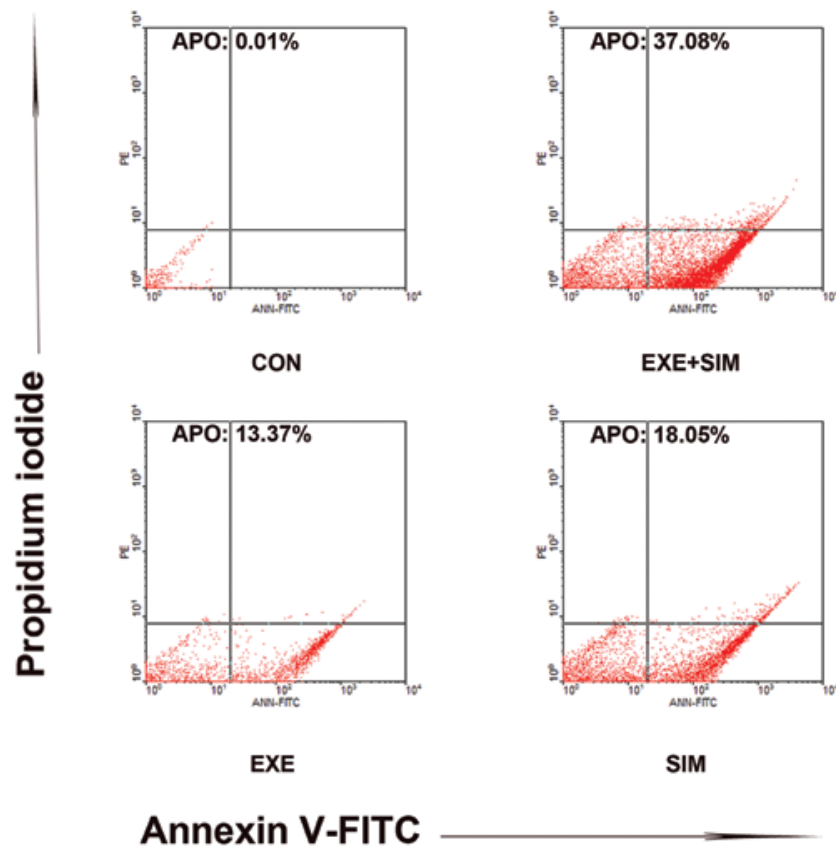

Figure 4. Effects of exemestane and simvastatin on the rate of cell apoptosis. The effects of each single drug and their combination on the induction of apoptosis in MCF-7 human breast cancer cells were determined by flow cytometry. After $72 \mathrm{~h}$ of treatment, adherent and floating cells were collected and incubated with Annexin V and propidium iodide. Apoptotic rates were determined using the computer software Cell Quest. CON, control; EXE, exemestane; SIM, simvastatin; EXE+SIM, concurrent administration; FITC, fluorescein isothiocyanate.

Effects of simvastatin and exemestane treatment on the expression levels of $\mathrm{Bcl}-2$ and Bax. To further evaluate the potential synergistic mechanisms of exemestane and simvastatin, the protein expression levels of Bcl-2 and Bax in MCF-7 cells were detected by western blotting. Combined treatment of exemestane with simvastatin resulted in a marked reduction in the protein expression levels of $\mathrm{Bcl}-2$, and an increase in the expression levels of Bax, as compared with those in the control cells and those treated with simvastatin or exemestane alone (Fig. 6).

\section{Discussion}

Breast cancer is a hormone-dependent disease that relies on the mitogenic effects of estrogen to drive carcinogenesis. AIs are currently used as the standard first-line treatment to significantly reduce the risk of recurrence for postmenopausal females with ER+ metastatic breast cancer, as they have been proven to be more effective than tamoxifen (5). An open-label, randomized, phase III study conducted by the European Organisation for the Research and Treatment of Cancer reported a significant improvement in median progression-free survival and overall response rate for exemestane treatment, as compared with tamoxifen (27). However, despite the proven clinical efficacy of AIs in the treatment of breast cancer, de novo and acquired drug resistance often occurs and presents a major obstacle to successful therapy. In addition, patients with breast cancer treated with AIs have a higher incidence of AI-associated musculoskeletal symptoms. An International Standard Randomised Controlled Trial reported

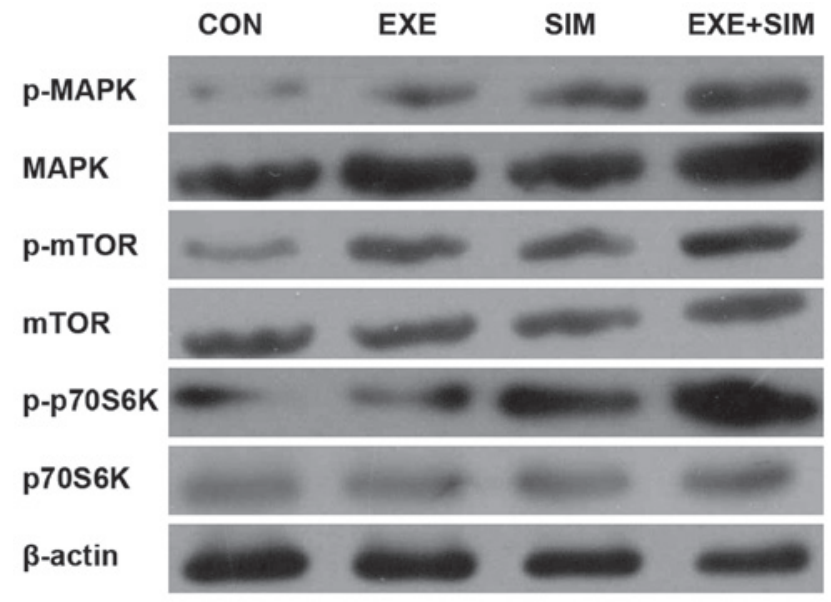

Figure 5. Effects of exemestane combined with simvastatin on the expression levels of MAPK and mTOR/p70S6 signaling-associated proteins in MCF-7 human breast cancer cells. The cells were treated with exemestane and simvastatin alone or in combination for $72 \mathrm{~h}$ at their $\mathrm{IC}_{50}$ concentration. The expression levels of MAPK and mTOR/p70S6 signaling-associated proteins were then analyzed by western blot analysis with corresponding antibodies. $\beta$-actin was used as a loading and normalization control. CON, control; EXE, exemestane; SIM, simvastatin; EXE+SIM, concurrent administration; MAPK, mitogen-activated protein kinase; mTOR, mammalian target of rapamycin; p, phosphorylated.

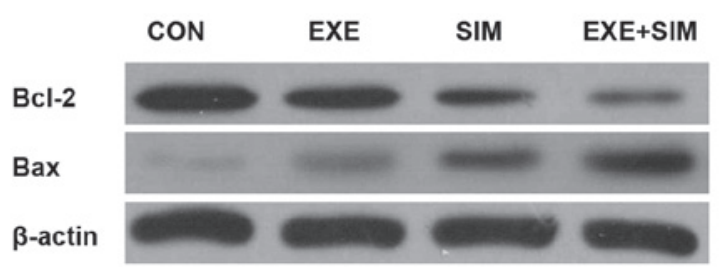

Figure 6. Effects of exemestane combined with simvastatin on the protein expression levels of Bcl-2 and Bax in MCF-7 human breast cancer cells. The Bcl-2 and Bax family have an important role in the regulation of apoptosis, proliferation and the invasion of tumor cells. MCF-7 cells were treated with exemestane, simvastatin or a combination of both for $72 \mathrm{~h}$, using their $\mathrm{IC}_{50}$ concentrations. After treatment, the cells were harvested and lysed, and equal amounts of extracted protein were analyzed for Bcl-2 and Bax expression levels by western blotting. $\beta$-actin was used as a loading control. CON, control; EXE, exemestane; SIM, simvastatin; EXE+SIM, concurrent administration; Bcl-2, B-cell lymphoma 2; Bax, Bcl-2-associated X protein.

that females receiving exemestane experienced significantly higher rates of arthralgia (28). A previous study demonstrated that Aromasin $^{\circledR}$ (exemestane) combined with simvastatin was able to significantly increase bone mineral density, thus suggesting that simvastatin may have potential therapeutic application in the treatment of osteoporosis, to counterbalance the adverse effects of exemestane (29). Furthermore, numerous preclinical studies have shown that statins possess anti-proliferative, anti-angiogenic, anti-metastatic and pro-apoptotic properties in various types of cancer cell $(17,18)$. In addition, some epidemiological studies have also demonstrated that statins are associated with a lower incidence of invasive breast cancer, these findings suggest that statins may contribute to the primary prevention of breast cancer $(19,22)$. However, to the best of our knowledge, the effects of statins on endocrine therapies for patients with ER+ breast cancer have remained to be elucidated. Therefore, the present study investigated the 
efficacy of simvastatin, either alone or in combination with exemestane, on the MCF-7 ER+ breast cancer cell line. The results of the present study demonstrated that simvastatin enhanced the inhibitory effects of exemestane on the growth of ER+ breast cancer cells. When simvastatin was combined with exemestane, the concentration of exemestane required to inhibit cancer cell growth was significantly reduced. These results indicated the potential importance of combined treatment approaches for increasing the efficacy of exemestane, and lessening its associated side-effects.

Simvastatin is a widely used cholesterol-adjusting drug, which selectively inhibits HMG-CoA reductase, leading to decreased cholesterol biosynthesis. Population-based studies have demonstrated that treatment with simvastatin is associated with a substantial reduction in the risk of breast cancer $(19,22)$. Furthermore, the ex vivo tumor cell inhibition of simvastatin and its additive effects upon combination with cisplatin or docetaxel, provide the basis for epidemiological and clinical studies on statins, potentially directed toward co-medication in future treatment regimens (30). Cholesterol is a steroid hormone precursor, and the majority of cases of breast cancer are considered hormone responsive (31). Previous studies in genetic- or diet-induced hypercholesterolemic murine models have demonstrated an obvious association between high lipid levels and breast cancer progression (32). Furthermore, a primary metabolite of cholesterol, oxysterol 27-hydroxycholesterol, has been shown to promote the growth of ER+ breast cancer in in vivo models (33). Therefore, the reversal of these processes by the oral lipid-lowering drug simvastatin is an attractive anti-cancer strategy.

The results of the present study indicated that simvastatin and exemestane inhibited the proliferation of MCF-7 cells in a concentration-dependent manner. Combined treatment of simvastatin with exemestane for $72 \mathrm{~h}$ resulted in a marked increase in the inhibition of cell growth, as compared with treatment with exemestane or simvastatin alone. The present study also demonstrated an enhanced effect on cell cycle progression and apoptosis. MAPK is a key kinase that has an essential role in energy homeostasis and regulates processes associated with the development of cancer, including cell proliferation and survival (34,35), cell cycle arrest (34) and protein synthesis (35). Despite uncontrolled cellular proliferation in breast cancer, which is theoretically expected to create a large demand for cellular energy, there is histological evidence that phosphorylation of MAPK at Thr-172 is downregulated, particularly in tumors of high histological grade that are associated with axillary node metastasis (36). The MAPK signaling pathway not only promotes cell proliferation, but also induces cell apoptosis and is known to be upregulated in cancer cells (37). The main downstream effect of MAPK activation is the inhibition of the mTOR signaling pathways. Misirkic et al (38) reported that in simvastatin-treated glioma cells, inhibition of mTOR, and its substrate S6K1, resulted in the activation of the mTOR negative regulator MAPK. This result is concordant with the previously reported ability of statins to activate MAPK in hepatic and colorectal cancer cells in vitro (39), as well as to inhibit the Akt/mTOR signaling pathway in renal cancer cells (40). In the present study, combined treatment of exemestane with simvastatin caused a large decrease in the relative protein expression levels of
p-MAPK and decreased the expression levels of p-mTOR, as compared with those in the control or single-agent treatment groups. p70S6K is one of the main downstream effectors of the mTOR signaling pathway, and its activated form p-p70S6K was also shown to be inhibited in the present study. To the best of our knowledge, the present study was the first to examine the individual and combined effects of simvastatin and exemestane on MCF-7 ER+ breast cancer cells, and to investigate the underlying apoptotic and growth pathways involved.

The results of the present study also demonstrated that simvastatin and exemestane increased the expression levels of Bax and decreased the expression levels of $\mathrm{Bcl}-2$. The progression of cancer depends on the balance between pro-apoptotic proteins, such as Bax and anti-apoptotic proteins, such as Bcl-2 (15,41). Bcl-2 and Bax are key apoptosis regulators in numerous types of cells, which in response to treatment may lead to the activation of apoptosis (15). The results of the present study indicated that regulation of Bax and Bcl-2 protein expression may be involved in combination treatment-induced cell death.

In conclusion, the results of the present study indicated that simvastatin combined with exemestane may have synergistic effects on cell proliferation and induce cell cycle arrest and apoptosis of MCF-7 human breast cancer cells in vitro. The present study further confirmed that the synergistic effects of these two agents may involve the $\mathrm{Bax} / \mathrm{Bcl}-2$ apoptotic pathway and the MAPK/mTOR/p70S6K growth pathway. The anti-tumor effects of simvastatin are complex and remain to be fully elucidated; however, these findings provided direct evidence of its efficacy on ER+ breast cancer cells when used in combination with exemestane. Futhermore, the results of the present study suggested that the combination of simvastatin and exemestane may be a potential therapeutic strategy used to treat breast cancer; however, the synergistic effects of these two drugs require a large-scale clinical trial for further validation.

\section{Acknowledgements}

The present study was supported by grants from the Anhui Provincial Science and Technology Agency Foundation of China (grant nos. 1301042214, 12070403072 and KJ2012A157). The authors would also like to thank Mrs. YuanYuan, The Central Laboratory of Binhu Hospital, The Third Affiliated Hospital of Anhui Medical University (Anhui, China), for experimental instruction. The authors declare that they have no conflict of interest.

\section{References}

1. Ferlay J, Parkin DM and Steliarova-Foucher E: Estimates of cancer incidence and mortality in Europe in 2008. Eur J Cancer 46: 765-781, 2010.

2. Chen S: An "omics" approach to determine the mechanisms of acquired aromatase inhibitor resistance. OMICS 15: 347-352, 2011.

3. Jankowitz RC and Davidson NE: Adjuvant endocrine therapy for breast cancer: how long is long enough? Oncology (Williston Park) 27: 1210-1216, 2013.

4. Campagnoli C, Pasanisi P, Castellano I, Abba C, Brucato T and Berrino F: Postmenopausal breast cancer, androgens, and aromatase inhibitors. Breast Cancer Res Treat 139: 1-11, 2013. 
5. Riemsma R, Forbes CA, Kessels A, et al: Systematic review of aromatase inhibitors in the first-line treatment for hormone sensitive advanced or metastatic breast cancer. Breast Cancer Res Treat 123: 9-24, 2010.

6. Li Z, Gu X, Tong J, et al: A meta-analysis of internal mammary lymph node metastasis in breast cancer patients. Onkologie 36 : 747-752, 2013.

7. Morris PG, McArthur HL and Hudis CA: Therapeutic options for metastatic breast cancer. Expert Opin Pharmacother 10: 967-981, 2009.

8. Rizzoli R, Body JJ, De Censi A, Reginster JY, Piscitelli P, Brandi ML; European Society for Clinical and Economical aspects of Osteoporosis and Osteoarthritis (ESCEO): Guidance for the prevention of bone loss and fractures in postmenopausal women treated with aromatase inhibitors for breast cancer: an ESCEO position paper. Osteoporos Int 23: 2567-2576, 2012.

9. Brodie A and Sabnis G: Adaptive changes result in activation of alternate signaling pathways and acquisition of resistance to aromatase inhibitors. Clin Cancer Res 17: 4208-4213, 2011

10. Wong WW, Dimitroulakos J, Minden MD and Penn LZ: HMG-CoA reductase inhibitors and the malignant cell: the statin family of drugs as triggers of tumor-specific apoptosis Leukemia 16: 508-519, 2002.

11. Superko HR, Momary KM and Li Y: Statins personalized. Med Clin North Am 96: 123-139, 2012.

12. Goldstein JL and Brown MS: Regulation of the mevalonate pathway. Nature 343: 425-430, 1990.

13. Liao JK: Isoprenoids as mediators of the biological effects of statins. J Clin Invest 110: 285-288, 2002.

14. Wejde J, Blegen $\mathrm{H}$ and Larsson O: Requirement for mevalonate in the control of proliferation of human breast cancer cells. Anticancer Res 12: 317-324, 1992.

15. Spampanato C, De Maria S, Sarnataro M, et al: Simvastatin inhibits cancer cell growth by inducing apoptosis correlated to activation of Bax and down-regulation of BCL-2 gene expression. Int J Oncol 40: 935-941, 2012

16. Kochuparambil ST, Al-Husein B, Goc A, Soliman S and Somanath PR: Anticancer efficacy of simvastatin on prostate cancer cells and tumor xenografts is associated with inhibition of Akt and reduced prostate-specific antigen expression. J Pharmacol Exp Ther 336: 496-505, 2011.

17. Campbell MJ, Esserman LJ, Zhou Y, et al: Breast cancer growth prevention by statins. Cancer Res 66: 8707-8714, 2006

18. Cho SJ, Kim JS, Kim JM, Lee JY, Jung HC and Song IS: Simvastatin induces apoptosis in human colon cancer cells and in tumor xenografts, and attenuates colitis-associated colon cancer in mice. Int J Cancer 123: 951-957, 2008.

19. Nielsen SF, Nordestgaard BG and Bojesen SE: Statin use and reduced cancer-related mortality. N Engl J Med 367: 1792-1802, 2012

20. Guterres FA, Martinez GR, Rocha ME and Winnischofer SM Simvastatin rises reactive oxygen species levels and induces senescence in human melanoma cells by activation of p53/p21 pathway. Exp Cell Res 319: 2977-2988, 2013.

21. Cheng WH, Ho WY, Chang CF, et al: Simvastatin induces a central hypotensive effect via Ras-mediated signalling to cause eNOS up-regulation. Br J Pharmacol 170: 847-858, 2013.

22. Ahern TP, Pedersen L, Tarp M, et al: Statin prescriptions and breast cancer recurrence risk: a Danish nationwide prospective cohort study. J Natl Cancer Inst 103: 1461-1468, 2011

23. Hu LX, Du YY, Zhang Y and Pan YY: Synergistic effects of exemestane and aspirin on MCF-7 human breast cancer cells. Asian Pac J Cancer Prev 13: 5903-5908, 2012.
24. Budman DR, Calabro A, Wang LG, et al: Synergism of cytotoxic effects of vinorelbine and paclitaxel in vitro. Cancer Invest 18 : 695-701, 2000.

25. Budman DR and Calabro A: In vitro search for synergy and antagonism: evaluation of docetaxel combinations in breast cancer cell lines. Breast Cancer Res Treat 74: 41-46, 2002.

26. Chou TC and Talalay P: Quantitative analysis of dose-effect relationships: the combined effects of multiple drugs or enzyme inhibitors. Adv Enzyme Regul 22: 27-55, 1984.

27. Paridaens RJ, Dirix LY, Beex LV, et al: Phase III study comparing exemestane with tamoxifen as first-line hormonal treatment of metastatic breast cancer in postmenopausal women: the European Organisation for Research and Treatment of Cancer Breast Cancer Cooperative Group. J Clin Oncol 26: 4883-4890, 2008.

28. Sestak I, Cuzick J, Sapunar F, et al; ATAC Trialists' Group: Risk factors for joint symptoms in patients enrolled in the ATAC trial: a retrospective, exploratory analysis. Lancet Oncol 9: 866-872, 2008

29. Chen SH, Chou FF and Ko JY: The use of simvastatin with aromasin in an ovariectomized rat model: effects on the skeletal system. Chang Gung Med J 33: 509-514, 2010.

30. Stoehr M,MozetC,Boehm A,Aigner A, Dietz A and Wichmann G: Simvastatin suppresses head and neck squamous cell carcinoma ex vivo and enhances the cytostatic effects of chemotherapeutics. Cancer Chemother Pharmacol 73: 827-837, 2014.

31. Danilo C and Frank PG: Cholesterol and breast cancer development. Curr Opin Pharmacol 12: 677-682, 2012.

32. Llaverias G, Danilo C, Mercier I, et al: Role of cholesterol in the development and progression of breast cancer. Am J Pathol 178: 402-412, 2011.

33. Nelson ER, Wardell SE, Jasper JS, et al: 27-Hydroxycholesterol links hypercholesterolemia and breast cancer pathophysiology. Science 342: 1094-1098, 2013.

34. Imamura K, Ogura T, Kishimoto A, Kaminishi $M$ and Esumi H: Cell cycle regulation via p53 phosphorylation by a 5'-AMP activated protein kinase activator, 5-aminoimidazole-4-carboxamide-1-beta-D-ribofuranoside, in a human hepatocellular carcinoma cell line. Biochem Biophys Res Commun 287: 562-567, 2001

35. Jones RG, Plas DR, Kubek S, et al: AMP-activated protein kinase induces a p53-dependent metabolic checkpoint. Mol Cell 18: 283-293, 2005.

36. Hadad SM, Baker L, Quinlan PR, et al: Histological evaluation of AMPK signalling in primary breast cancer. BMC Cancer 9: 307, 2009.

37. Bühler S and Laufer SA: p38 MAPK inhibitors: a patent review (2012 - 2013). Expert Opin Ther Pat 24: 535-554, 2014.

38. Misirkic M, Janjetovic K, Vucicevic L, et al: Inhibition of AMPK-dependent autophagy enhances in vitro antiglioma effect of simvastatin. Pharmacol Res 65: 111-119, 2012

39. Yang PM, Liu YL, Lin YC, Shun CT, Wu MS and Chen CC: Inhibition of autophagy enhances anticancer effects of atorvastatin in digestive malignancies. Cancer Res 70: 7699-7709, 2010.

40. Woodard J, Sassano A, Hay $N$ and Platanias LC: Statin-dependent suppression of the Akt/mammalian target of rapamycin signaling cascade and programmed cell death 4 up-regulation in renal cell carcinoma. Clin Cancer Res 14: 4640-4649, 2008.

41. Jaafar H, Abdullah S, Murtey MD and Idris FM: Expression of Bax and Bcl-2 in tumour cells and blood vessels of breast cancer and their association with angiogenesis and hormonal receptors. Asian Pac J Cancer Prev 13: 3857-3862, 2012. 